\title{
Which battery of cardiovascular autonomic function tests?
}

\section{Comment}

\author{
D.J. Ewing \\ University Department of Medicine, Royal Infirmary, Edinburgh, Scotland, UK
}

Numerous investigators have set out on the quest for the 'Holy Grail' of a battery of perfect cardiovascular autonomic function tests. None have reached that goal! The debate between researchers, in accepting that no 'perfect' test exists, concerns which tests are most useful, which are easiest to perform, and what are the best techniques and measurements to apply [1]. Drs Ryder and Hardisty have brought out some of these issues in their article. They rightly point out that standardisation is important, but question whether the Edinburgh battery of tests is as good as the test protocol proposed by Dr. O'Brien and colleagues [2]. I must take issue with them on some points of detail.

The differences between the Edinburgh and O'Brien batteries are more that of technical aspects rather than the actual tests involved, except in one important respect. The O'Brien battery, in my view, duplicates one test (heart rate variation) under two different guises, namely "maximumminimum' heart rate, and standard deviation of RR interval. In fact, these are different ways of measuring the same thing, and therefore only one of the tests should be used. We showed some years ago that maximum-minimum heart rate was more practical for general clinical use than standard deviation [3].

With regard to differences in technique between the Edinburgh and O'Brien batteries, Ryder states that the heart rate changes evoked by a single deep breath are greater than those evoked by repeated breaths. He has not quoted our own work on this [4], where we showed that in only $29 \%$ of normal subjects and $17 \%$ of diabetic patients the first deep breath consistently produced the largest heart rate variation; and that the between-test variability was smaller with repeated deep breaths. It has always seemed preferable to me to average several breaths to reduce the between-test variability as much as possible. The same argument can be applied to performing several Valsalva manoeuvres, rather than just one. Patients sometimes perform the first manoeuvre incorrectly, and this would be wrongly classified as 'abnormal' if only one test was used. Whether one measures the shortest beat during or immediately after the Valsalva manoeuvre makes, in my experience, only a slight difference to the Valsalva ratio, and certainly is not relevant in an abnormal heart rate response to the Valsalva manoeuvre, which is so obviously different from a normal response.

Ryder and Hardisty make a very valid point about the lying-to-standing 30:15 ratio, which is really the maximum-minimum ratio, as there is some slight variation in normal subjects about the points of shortest and longest RR interval after standing up. However, there is a physiological misconception in the O'Brien battery where the count of beats is taken from the time when the subject is actually standing up. RR interval shortening occurs immediately a subject starts to stand up, irrespective of whether this is done quickly or slowly [5]. It is not surprising, therefore, that among O'Brien's normal subjects some curious $30: 15$ ratios were obtained. The question of movement artifact during the lying-to-standing test is irrelevant. This usually occurs only for the 3 or 4 beats while the subject is actually moving, and makes no difference to the heart beats later on around beats 15 and 30. Can the O'Brien modifications be performed more quickly? Most of the time in any test is taken up by putting on electrodes and preparing the patient. While I would agree with Ryder that measurement of ECG strips is time-consuming, he also states that 'computer-assisted systems are readily available', which of course produce almost instantaneous answers. Our own Autocaft system was purpose-built to fit the Edinburgh test battery.

Age-related normal values are, of course, very relevant. However, while almost all observers have found that the heart rate response to deep breathing and standing up are age-related, there have been differences with regard to the Valsalva response. Some observers have found that age is significant, whereas others like ourselves have not [6]. Should one use age-related normal ranges? This partly depends on the use to which the tests are applied. If the tests are being used for research purposes, then I agree entirely with Ryder and Hardisty, and indeed with most other investigators, that age-matched normal control subjects should be used. If, on the other hand, they are being used for diagnostic purposes, then in my experience there 
are lower limits of normal, which apply at any age. What a clinician requires is a clear statement, based on several tests, as to whether autonomic neuropathy is present, and its severity. There is a danger in being too precise in individual tests at the 'borderline' between normal and abnormal, particularly in view of the known test-test variability.

Drs. Ryder and Hardisty have provided a stimulating challenge to us all to look constructively at the tests we currently use to diagnose autonomic neuropathy. Although I might be said to be biased, I am not convinced by their arguments that the O'Brien battery offers any significant advantage over the Edinburgh tests, which I will continue to use!

\section{References}

1. Ewing DJ (1988) Recent advances in the non-invasive investigation of diabetic autonomic neuropathy. In: Bannister R (ed) Autonomic Failure. A textbook of clinical disorders of the autonomic nervous system, 2nd edn. Oxford University Press, Oxford New York Tokyo, pp 667-689

2. O'Brien IAD, O'Hare P, Corrall RJM (1986) Heart rate variability in healthy subjects: effect of age and the derivation of normal range for tests of autonomic function. $\mathrm{Br}$ Heart J 55: 348-354

3. Ewing DJ, Borsey DQ, Bellavere F, Clarke BF (1981) Cardiac autonomic neuropathy in diabetes: comparison of measures of $\mathrm{R}-\mathrm{R}$ interval variation. Diabetologia $21: 18-24$

4. Espi F, Ewing DJ, Clarke BF (1982) Testing for heart rate variation in diabetes: single or repeated deep breaths? Acta Diabet Latina 19: 177-181

5. Ewing DJ, Hume L, Campbell IW, Murray A, Neilson JMM, Clarke BF (1980) Autonomic mechanisms in the initial heart rate response to standing. J Appl Physiol 49: 809-814

6. Ewing DJ, Martyn CN, Young RJ, Clarke BF (1985) The value of cardiovascular autonomic function tests: 10 years experience in diabetes. Diabetes Care 8: 491-498

\section{Dr. D.J.Ewing}

University Department of Medicine

Royal Infirmary

Edinburgh EH3 9YW

Scotland, UK 